\title{
Insights from advancements and pathbreaking research on the minimally invasive treatment of atrial fibrillation
}

\author{
Anna Witkowska^, Piotr Suwalski^ \\ Department of Cardiac Surgery, Central Clinical Hospital of the Ministry of Interior and Administration, Centre of Medical Postgraduate Education, \\ Warsaw, Poland \\ Contributions: (I) Conception and design: P Suwalski; (II) Administrative support: All authors; (III) Provision of study materials or patients: All \\ authors; (IV) Collection and assembly of data: A Witkowska; (V) Data analysis and interpretation: None; (VI) Manuscript writing: All authors; (VII) \\ Final approval of manuscript: All authors. \\ Correspondence to: Anna Witkowska. Department of Cardiac Surgery, Central Clinical Hospital of the Ministry of Interior and Administration, Centre \\ of Medical Postgraduate Education, Warsaw, Poland. Email: vera@rallywitkowscy.pl.
}

\begin{abstract}
Atrial fibrillation (AF) remains the most common cardiac arrhythmia with increasing prevalence in developed and aging countries. Pharmacological antiarrhythmic therapy has low effectiveness and is limited by its toxicity. Developed in 1987 by James Cox surgical ablation of AF called MAZE procedure was very effective, but due to its invasiveness and complexity was not widely adopted. Landmark research done by Haissaguerre in 1998 initiated a new approach for treatment namely percutaneous catheter ablation, which remains a class I/A indication in symptomatic paroxysmal AF refractory to optimal medical therapy. However, its efficacy in patients with persistent atrial fibrillation (PSAF) is far from satisfactory. Recent advancements in devices and techniques of minimally invasive surgical ablation show very good results in the treatment of PSAF. Current guidelines equate surgical with catheter ablation within the scope of efficacy indicating that both may be considered as an effective and safe treatment option for patients with persistent forms of arrhythmia. The higher efficacy of surgical ablation was confirmed at a 7-year follow-up of FAST trial with recurrence rate as high as $87 \%$ in catheter arm compared with $56 \%$ in thoracoscopic ablation arm. A new concept of the invasive treatment of AF consisting of combined surgical (epicardial) and electrophysiological (endocardial) was introduced in 2009. Recently experts' opinions and published data suggest that the proper hybrid treatment consisting of a planned combination of surgical and catheter ablation may give even better results. One of the most invaluable benefits of surgical ablations is the possibility of concomitant occlusion of the left atrial appendage. Recently good results have been reported for the novel epicardial clip for closing the left atrial appendage, which is placed in the deployment loop on a disposable holder.
\end{abstract}

Keywords: Ablation; atrial fibrillation (AF); minimally invasive surgery; hybrid approach

Submitted May 10, 2020. Accepted for publication Oct 14, 2020.

doi: $10.21037 /$ jtd-20-1876

View this article at: http://dx.doi.org/10.21037/jtd-20-1876

\section{Introduction}

Atrial fibrillation (AF) is the most common cardiac arrhythmia with increasing prevalence in developed and aging countries. It occurs in $3 \%$ of the population over 20 - year and even $9 \%$ in those over 80 -year. Estimates anticipate that by $2030,14-17$ million patients in the European Union will develop AF and 120,000-215,000 patients will be diagnosed every year (1). An increasing lifespan with the advancement in medical care may significantly contribute

^ ORCID: Piotr Suwalski, 0000-0003-2670-4164; Anna Witkowska, 0000-0001-8188-0422. 
to a greater incidence of AF, especially in cohort with hypertension, structural heart disease, obesity, diabetes, or endocrinological disorders. The main issue in AF treatment is the adverse events as it is associated with a 5 -fold increased risk of thromboembolic events, 2-fold increased risk of myocardial infarction, repeated hospitalizations, and worse quality of life (1-4). These factors raise mortality and the economic and social cost of care of this group of patients (1,5-7).

Pharmacological antiarrhythmic therapy has low effectiveness and is limited by its toxicity, and recently there were no significant improvements in this field $(8,9)$. The introduction of the novel oral anticoagulants (NOACs) improved compliance and safety of the therapy mainly due to a reduction in hemorrhagic complications $(1,10)$. Nevertheless, the most important measure in stroke prevention remains the stable sinus rhythm restoration.

Developed in 1987 by James Cox surgical ablation of AF called MAZE procedure was very effective, but due to its invasiveness and complexity was not widely adopted $(11,12)$. In subsequent years it was modified by its author in terms of lesions sets establishing the MAZE III procedure, which to this very day is the gold standard in surgical AF ablation (13). Further evolvement changed the way to create a transmural scar and enable minimalization of surgical access, which universalized and popularized the procedure $(3,14)$. As a new standard regarded is the endocardial biatrial lesion set performed with bipolar radiofrequency and/or cryoablation through right minithoracotomy called MAZE IV $(15,16)$. Recently surgical ablation in patients with arrhythmia undergoing other cardiac operations is recommended in class IIaA and the scars should be performed with radiofrequency energy or cryothermy (3). Landmark research done by Haissaguerre in 1998 initiated a new approach for the treatment and gave the rudiments of percutaneous catheter ablation (17) which is now the basic treatment for paroxysmal AF refractory to optimal medical therapy (class IA indication) (1). However, for stand-alone persistent forms of atrial fibrillation (PSAF) results of this treatment remain far from satisfactory (18-20). In 2019 Berger et al. published a meta-analysis of 60 papers presenting results of invasive treatment of persistent $\mathrm{AF}$ published from 2006 to 2018. The analysis showed that surgical minimally invasive procedures have a higher success rate in sinus rhythm restoration, however with more adverse events (21). Recent advancements in techniques of minimally invasive surgical ablation show very good efficacy in the treatment of PSAF (22). In guidelines published by the European Society of Cardiology in 2016 surgical and catheter ablation should be considered as a therapeutic option for persistent and long-standing persistent $\mathrm{AF}$ ( $\mathrm{IIaC}$ ) indicating that both types of treatment are effective and safe $(1,19,23,24)$. Moreover, published data indicate that success rates of catheter procedures decline with a given time and are lower than $50 \%$ in 1 year (25). Frequently patients undergo multiple ablations what increases the exposition to radiation, risk of complications, and healthcare costs. Surgical ablation offers a higher success rate, but its higher invasiveness, chest incisions, and dissections around the heart limit widespread acceptance. In the last update of the guidelines of management of AF published by the European Society of Cardiology (ESC) and by the Society of Thoracic Surgeons recommend that surgical ablation as a stand-alone procedure should be considered in patients who have failed a previous catheter ablation (IIaB) or with refractory to AAD therapy symptomatic arrhythmia as a patient choice in the level of evidence $\mathrm{IIaC}(1,3)$. However, the surgical procedure should be performed with minimally invasive techniques in an experienced center (IIaC) (1).

\section{The key concepts}

J. Cox proposed the concept of surgical ablation based on the surgically made pathway for the electric impulse from the sinus node to the atrioventricular node (12). Those pathways were made by cutting and sewing the muscle of the left and right atria, that is why it is called "the Maze" procedure. Although extremely effective, it is technically challenging while requires a full sternotomy, cardiopulmonary bypass with aortic cross-clamping, and cardiac arrest. The main complications, that occurred quite frequently were bleeding and complete atrioventricular block. Advancement in minimally invasive techniques $(26,27)$ allowed for the reappearance of the surgical ablation with "maze-pattern" lines through a minimal surgical incision (28). The next step was the introduction of the offpump epicardial techniques, however, the main problem was the creation of transmural lesions and effectiveness in sinus rhythm restoration. Numerous energy sources have been tested, but recently only radiofrequency and cryothermy confirmed the effectiveness and are acceptable (3). Constraints associated with limited access impose modifications of the original MAZE lesion set, however, the essentials of the concept remained the same: (I) pulmonary vein isolation; (II) modification of the substrate; (III) addressing the left atrial appendage (LAA) whenever 
possible.

This review aims to present medical professionals emerging options of treatment of stand-alone PSAF and long-standing PSAF which benefits from new minimally invasive techniques, namely:

* Stand-alone surgical epicardial ablation with bipolar radiofrequency energy and exclusion of the left atrial appendage via thoracoscopic access;

* Hybrid approach as a planned strategy of minimally invasive surgical ablation followed by transcatheter electrophysiological control after the blanking period

* The hybrid convergent procedure with connected epicardial and endocardial ablation for complete extended lesion pattern.

\section{The stand-alone minimally invasive surgical procedure}

The first step towards reducing the invasiveness of the surgical approach was done by Randal Wolf in 2005. He performed the off-pump epicardial isolation of the right and left pulmonary veins through bilateral mini-thoracotomies with a bipolar radiofrequency device (29). The "Wolf procedure" included bilateral antrum isolation with partial cardiac denervation. The next step was a totally endoscopic approach that enforced the oversimplification of the concept of a "box-lesion" as a single circular lesion around all four pulmonary veins including the posterior wall of the LA. The lesion was created with unipolar radiofrequency devices with an ablating part placed on the inner side of a single tube. $(30,31)$. However, separating the pericardial reflections or superior vena cava (SCV) from the right pulmonary artery requires endoscopic skills and may lead to laceration and bleeding.

This procedure became widely accepted, but in longer observation effectiveness in SR restoration was moderate and it was dedicated mainly for the treatment of paroxysmal $\mathrm{AF}$ which was already taken over by less invasive percutaneous techniques. Nevertheless, the idea of a surgical "box-lesion" was further developed and in 2011 Muneretto et al. presented a partly hybrid approach using an RF device (Estech, Cobra Adhere XL, AtriCure, Inc., West Chester, Ohio, USA) to create a primary "box-lesion" via right-sided thoracoscopic access with concomitant implantation of a continuous monitoring rhythm device (Reveal XT; Medtronic, Minneapolis, MN USA) (22). Other modification named "Brescia Lesion Set" involved the addition of three lesions on the lateral wall of the right atrium. This approach resulted in a success rate exciding $85 \%$, and additionally, as an emerging conception of hybrid treatment in patients with recurrences percutaneous catheter-based endocardial ablation was performed (14,32).

At the same time, Wolf's procedure was modified within the scope of the lesion pattern and converted to the videoassisted totally endoscopic ablation. The extent of the ablation was broadened and included not only pulmonary veins isolation but also the line to the mitral or aortic trigones, ganglionated plexi ablation, the obliteration of ligament of Marshall, and left atrial appendage exclusion (33). Lesions could be done through thoracoscopic access with dedicated bipolar devices manufactured by AtriCure (AtriCure, Inc., West Chester, Ohio, USA). This system was also used in the surgical arm of the Atrial Fibrillation Catheter Ablation Versus Surgical Ablation Treatment (FAST) (34,35).

\section{The FAST trial}

The Atrial Fibrillation Catheter Ablation Versus Surgical Ablation Treatment trial was the first prospective randomized clinical trial conducted to compare catheter and surgical ablation (34). The study included 125 patients randomized into two treatment arms. The higher efficacy of surgical ablation was confirmed at 1 year $(65 \%$ vs. $36 \%)$ as well as in a longer, mean 7-year follow-up with recurrence rate as high as $87 \%$ in catheter arm compared with $56 \%$ in thoracoscopic ablation arm (35). It is noteworthy that $23 \%$ of included patients had paroxysmal AF, and the continuation of AAD did not influence the results (78\% in surgical vs. $42 \%$ in catheter arm). This was the first systemic comparison which showed superior results of surgical ablation to catheter one in receiving freedom from left atrial arrhythmias in long-term observation. Although observed periprocedural adverse event rate was significantly higher in the surgical arm, most of them were minor and the composite endpoint of mortality, myocardial infarction, or cerebrovascular event was equal in both arms through all follow-up (34,35). Regrettably, the most remembered by the public was the number of periprocedural complications, not the success rate.

In a published in 2016 meta-analysis Phan et al. confirmed better results of surgical minimally invasive ablation for sinus rhythm restoration in comparison with catheter ablation (36). For patients with paroxysmal AF success rate was $82 \%$ for surgical procedure $v s .62 .5 \%$ for 


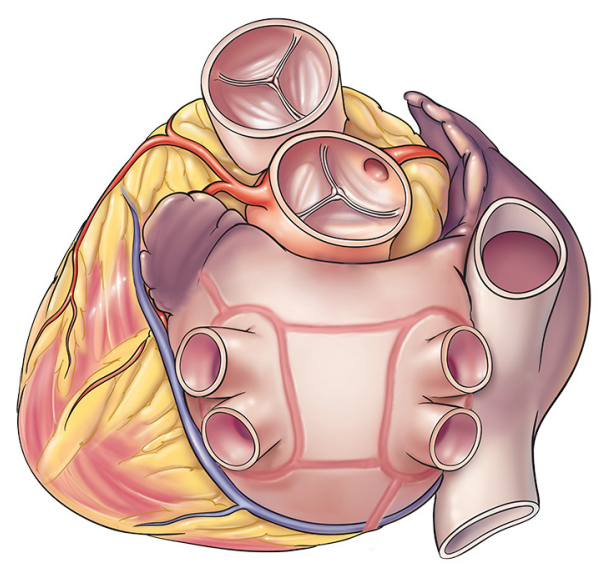

Figure 1 Left atrial lesion set for totally thoracoscopic ablation. The "Dallas" lesion. Courtesy: AtriCure.

catheter one and in patients with persistent $\mathrm{AF}$ the results were $74.4 \%$ vs. $51.1 \%$ respectively in 12 -month follow up (36). Recently there is an ongoing new randomized international multicenter trial comparing interventional treatment strategies in symptomatic patients with drugrefractory PSAF-CEASE-AF. The study has two armsone is a multiple catheter ablation and the second is a hybrid approach with totally thoracoscopic surgical ablation with left atrial appendage closing followed by electrophysiological examination 90-180 days later. In the time of publishing this article, the study was during the interim analysis.

\section{The totally thoracoscopic ablation procedure}

The totally thoracoscopic ablation of AF is an off-pump procedure employing bilateral access and extended lesion set which include PVI, connecting lines on the posterior wall of LA, a line to the non-coronary aortic sinus („trigonal” line) recently replaced with a line from left PVI to the LAA, the ganglionated plexi (GP) ablation, and the LAA exclusion (Figure 1). The procedure is performed with AtriCure (AtriCure, Inc., West Chester, Ohio, USA) system utilizing the bipolar radiofrequency energy.

The procedure was described in detail previously (37). In summary, trans-esophageal echocardiography is mandatory to rule out thromboembolic material in the left atrium. Thoracoscopic ports are placed through the 4th and 6th intercostal spaces in the midaxillary line. The working space is created with $\mathrm{CO} 2$ insufflation. First, the

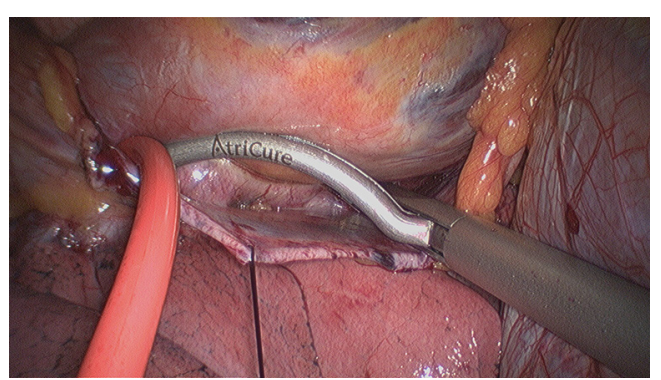

Figure 2 Standalone surgical ablation of AF. Isolator Synergy Clamps are placed around the right pulmonary veins. The view from the endoscopic camera.

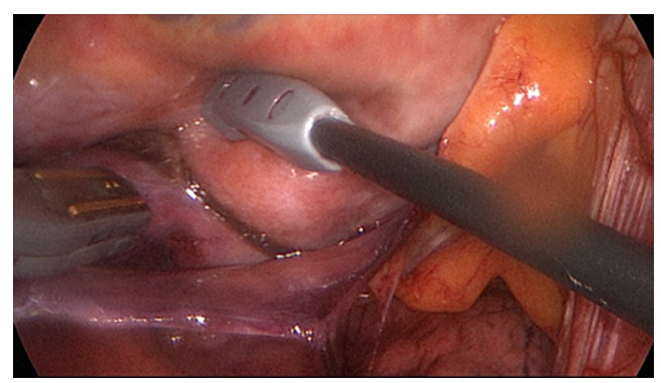

Figure 3 Standalone surgical ablation of AF. Clearly visible lesion separating right pulmonary veins from the left atrium. Ablation of ganglionated plexi with the unipolar device.

right-sided ablation is performed. The pulmonary veins and Waterstone's Groove (on the right side) are exposed, the oblique and transverse pericardial sinuses are opened and pulmonary veins are encircled with the AtriCure Lumitip Dissector (AtriCure, Inc., West Chester, Ohio, USA) to guide Isolator Synergy Clamp (AtriCure, Inc., West Chester, Ohio, USA) (Figure 2), the proper ablating device for PVI at the antrum of the veins (18). On the right side the identification and ablation of GP are performed (Figure 3) and from both sides, linear lesions with Isolator Linear Pen (AtriCure, Inc., West Chester, Ohio, USA) on the posterior wall of the left atrium connecting the right and left PVI lines. On the left side also the ligament of Marshall is addressed and left atrial appendage is excluded with a GI stapler or with dedicated epicardial clip AtriClip ${ }^{\circledR}$ PRO LAA Exclusion System (Atriclip, Atricure, Dayton, $\mathrm{OH}, \mathrm{USA})$. The obligatory endpoint of each ablation is the confirmation of bidirectional acute conduction block across pulmonary veins isolation defined as the absence of sensed atrial potentials in the PVs and pacing conduction to the atria from PVs in patients in sinus rhythm. 


\section{The hybrid approach}

A hybrid approach to AF treatment as defined by the ESC in 2016 guidelines comprises only combination antiarrhythmic drug therapy with catheter ablation (1). Such an understanding has quite a lot of drawbacks, as results are still far from satisfactory and patients do not avoid adverse events of AAD. Recently even more experts' opinions and published data suggest that the proper hybrid treatment should consist of a planned combination of surgical and catheter ablation. Such an approach for the first time reported Mahapatra et al. presenting the result of a sequential hybrid procedure in patients with PASF or LPASF and enlarged left atrium (LA size: $52 \mathrm{~mm}$ ) who already failed at least one percutaneous catheter ablation (38). The analyzed procedure consisted of thoracoscopic epicardial surgical ablation with PVs and SVC isolation, box lesions, mitral line, ganglionated plexi ablation, ablation of the ligament of Marshal, and LAA exclusion, followed by an endocardial EP procedure $4.3 \pm 1.3$ days later. In their study, $86.7 \%$ of the patients were free of any atrial arrhythmias without $\mathrm{AAD}$ in a mean follow-up of $20.7 \pm 4.5$ months (38).

Other publications also affirm better results of combined treatment $(32,39)$. The explanation may be found in the paper of Nanthakumar et al. As minimally invasive or thoracoscopic surgical ablations are mainly designed to create a full set of transmural lesions, they showed that recurrences are still present and conduction across the intraoperatively confirmed lines occurs in follow-up (40). Bulava et al. prospectively performed endocardial electrophysiological mapping at $94( \pm 30)$ days after epicardial radiofrequency ablation. Although $75.7 \%$ of patients were in stable sinus rhythm and $61.4 \%$ off AAD, they found complete lines around PVI in only $68.6 \%$ and lines on the posterior left atrial wall were complete in only $22.9 \%$ of cases (41). Termination of conduction was achieved using focal applications providing that in $82.9 \%$ of patients arrhythmia was not induced, what shows that the gaps were focal. This paper provides very important insight into the underbellies of epicardial procedure giving the basis for improvement in equipment and surgical technique. Also, Bisleri et al. reported better results when the electrophysiological examination is scheduled a few weeks after thoracoscopic ablation. In his cohort sinus rhythm restoration was augmented from $82.9 \%$ to $92.6 \%$ after focal catheter ablation (32).

Better results of hybrid treatment were recently published by Asmundis et al. Their approach consisted of totally thoracoscopic bilateral RF ablation followed by simultaneous catheter ablation. However, the success rate after the initial simultaneous hybrid procedure was $67.2 \%$ at a mean follow-up of $23.1( \pm 14.1)$ months, the overall freedom from AF including the following redo RF procedures was as high as $79.7 \%(42)$. The timing and sequence of the stages of the hybrid procedure remains a matter of debate. Doing the whole procedure during one session lessens the risk of repeated hospitalization, anesthesia, and procedure at the price of prolonged procedural time. Whereas separating the procedure in two stages with a 1 to 3 months gap allows the epicardial lesions to heal and stabilize, what might uncover more conduction gaps and warrants the overall efficacy (42). Also, two previous studies on sequential hybrid ablation reported a high overall success rate of $87 \%$ and $91.6 \%$ without $A A D$, suggesting that maturation of the lesion might be warranted to ensure better outcomes $(32,33)$. These encouraging observations were the background of an ongoing randomized multicenter trial CEASE-AF described above.

The modification of the abovementioned techniques is a multidisciplinary procedure merging the surgical thoracoscopic epicardial ablation with electrophysiological endocardial completing of lines. The reported results of this combined approach are very promising, in particular in patients with $\operatorname{LSPAF}(22,43)$.

\section{The convergent procedure}

The idea of joint ablation procedure involving both surgeon (epicardial ablation) and electrophysiologist (endocardial ablation) was initiated in 2009 and is now referred to as the convergent procedure. It has been widely adopted with favorable outcomes published by many centers and now the concept "Convergent Approach/Procedure" defines the cooperation of electrophysiologists and cardiac surgeons as the AF-Heart Team in the complex treatment of AF (44-46). The convergent procedure was described previously $(26,37)$. As in every rhythm control measure in AF treatment preceding TEE is mandatory. The classical approach is trans-diaphragmatic with standard laparoscopic techniques. Through a small incision below the xiphoid process in the midline abdomen, the central tendon of the diaphragm is exposed for the Subtle Cannula to be inserted into the pericardium. The cannula is the passage for the endoscope and ablation probe EPi-Sense Coagulation Device (AtriCure, Inc., West Chester, Ohio, USA; previously nContact Surgical Inc.). The ablating device utilizes unipolar 
radiofrequency energy which is regulated by an algorithm based on tissue impedance. This part of the procedure protocol implies a comprehensive epicardial ablation pattern including isolation of the posterior wall of the LA, posterior and anterior sides of both pulmonary veins, ganglionated plexi ablation, and in case of favorable anatomy also ablation of the ligament of Marshall $(26,37)$. However, due to the anatomy of pericardial reflections, transdiaphragmatic access does not allow to reach superior aspects of both PVs and the inferior region of the right pulmonary vein. That is why to complete circumvention of the pulmonary veins the second part-endocardial ablation is mandatory. The use of an electro-anatomical mapping system gives the possibility to perform the isopotential map of the LA and identification and ablation of all areas with persistent conductivity. At the end of the whole procedure, electrical isolation is verified by rapid pacing. The patient may be extubated on the operating table and discharged home on postoperative day 2-4 (26,37). The drawback of the convergent procedure is the utilization of fluoroscopic guidance for both endo and epicardial stages to visualize the relation between the ablating electrode and the esophagus (26).

The Convergent Procedure is widely performed with good results and safety profile in published data $(37,45,47,48)$. The one-year effectiveness in sinus rhythm restoration is reported from $79 \%$ to $95 \%$ of treated patients (37). The complication rate varies between the centers and ranges from none to $11 \%$ with the most frequently reported being mild pericarditis. Less frequent were stroke and transient ischemic attack (TIA) within the first 30 days, esophageal fistulas, bleeding, and tamponade $(37,49)$. The very important point is that those two procedures remain unchanged in their design and performance. The differences occur in timing between them, which may vary from ablation done sequentially during the same procedure to 7 to 21 days apart (37).

Presently new subxiphoid access to pericardium directly under the sternum is investigated for feasibility and efficacy. The skin incision is similar to the one seen in convergent procedure, however, the abdominal cavity is not opened and the pericardial sack is reached above the diaphragm (47).

\section{Rhythm monitoring}

The novel guidelines of surgical treatment of AF published by the Society of Thoracic Surgeons in 2017 recommends rhythm monitoring 3, 6, 9, 12, and 24 months after the procedures, preferably with 24-hour
Holter monitoring (3). The recurrence is defined as any atrial arrhythmia longer than 30 seconds (50). Although in guidelines of the European Society of Cardiology there is no clear information on how patients should be monitored, especially after invasive treatment, it is recommended to perform longer period monitoring, such as a 7-day Holter or implantable loop recorders rather than sole 24-hour Holter. In the expert consensus of the International Society for Holter and Noninvasive Electrocardiology (ISHNE) and Heart Rhythm Society (HRS) published in 2017 rhythm monitoring is mandatory after ablation including ECG recordings at every visit (51). For patients with paroxysmal AF 24-hour Holter should be performed at 1 year, also event recording continuously or at the time of symptoms from 3 to 12 months following ablation may be considered. For persistent AF 24-hour Holter monitoring is recommended each 6 months and additionally event-driven ECG monitoring (51). Holter monitoring is the most common way form rhythm assessment, however, it is limited by a relatively brief duration (52). However, they allow the registration of only one lead and as they have limited storage capability (generally less than 1 hour) retain only the information pertaining to relevant arrhythmias that are automatically detected. This may yield some arrhythmias to be missed and render the interpretation of the ECG difficult (52). Another interesting option is patientactivated event and loop recorders which can be used for several weeks and allows to capture the arrhythmia during symptomatic events $(51,52)$.

\section{LAA exclusion}

One of the most invaluable benefits of surgical ablations is the possibility of concomitant occlusion of the left atrial appendage. Based on results from randomized studies mainly on transcatheter devices, the European Society of Cardiology recommends the closure of LAA in the prevention of thromboembolic events in class IIbB (1). As surgical endocardial closure with the suture showed a remarkably high rate of recanalization in follow up it is strictly recommended to use dedicated surgical devices (53). There are few devices for epicardial LAA closure which may be used via classical or minimally invasive procedures. The totally thoracoscopic access via the chest wall gives straightforward access to the left atrial appendage. After opening the pericardium above and parallel to the phrenic nerve its feasible to safely exclude the appendage under direct vision. For this purpose, it is possible to 


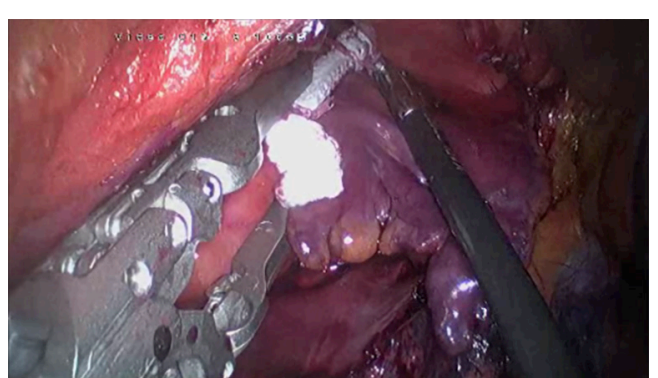

Figure 4 Totally thoracoscopic left atrial appendage occlusion with AtriClip PRO 2. The visible delivery system being withdrawn after clip release at the base of LAA. The view from the endoscopic camera.

use endoscopic Endo-GIA stapler with the reloads TriStaple (Medtronic, Minneapolis, USA), which shows very good properties in hermetic tissue squeezing with a sealed stapling line. However, due to the fact that the appendage is cut off there exists the risk of tear and life-threatening bleeding (54). Recently good results have been reported for the novel device AtriClip ${ }^{\circledR} \mathrm{PRO} 1$ and AtriClip ${ }^{\circledR} \mathrm{PRO} 2$ LAA Exclusion System (AtriClip, Atricure, Dayton, OH, USA) consisting of an automatic closing clip set on the deployment loop on a disposable holder (Figure 4). The clip is constructed from parallel titanium crossbars that equalize the force over the tissue trabeculations of the LAA. This ensures a sealed line at the base of the LAA orifice (55). Before final deployment, the clip can be reopened and adjusted in case of an unsatisfactory result. The family of AtriClip Devices includes the newest open-ended AtriClip PRO-V device for thoracoscopic delivery. Although during convergent ablation the LAA may be visualized with pericardioscopy, its occlusion via transdiaphragmatic access is impossible due to limited working space within the tubular cannula. However, it is possible to adopt minimally invasive thoracoscopic techniques and close the LAA through the left pleura. From this side, it is also possible to perform ablation on the anterior and superior parts of the left pulmonary veins, the ligament of Marshall, and the roof of the left atrium under direct vision. An alternative is percutaneous closure of LAA with either endocardial or hybrid devices (37).

\section{Final remarks}

Intensive advancement in minimally invasive surgical ablation techniques paved the way to overcome the main obstacle for widespread acceptance of this treatment of
AF. The high rate of stable sinus rhythm restoration in a few years follow-up after classical techniques are now reproduced with safer and more surgeon friendly energies and devices. There is increasing number of data pointing out a significant decline in the effectiveness of catheter ablation of PSAF in longer than the one-year follow-up. And as increasing number of patients require multiple catheter procedures there is a vast territory for minimally invasive, safe, and effective surgical treatment options for the most demanding patients. When coupled with transcatheter procedures in hybrid strategies they have the potential to reach better outcomes and patient satisfaction, along with the appropriate use of limited institutional resources.

\section{Acknowledgments}

Funding: None.

\section{Footnote}

Provenance and Peer Review: This article was commissioned by the Guest Editors (Jason Ali and Yasir Abu-Omar) for the series "Minimally Invasive Cardiac Surgery" published in Fournal of Thoracic Disease. The article has undergone external peer review.

Conflicts of Interest: Both authors have completed the ICMJE uniform disclosure form (available at http://dx.doi. org/10.21037/jtd-20-1876). The series "Minimally Invasive Cardiac Surgery" was commissioned by the editorial office without any funding or sponsorship. PS reports personal fees from Atricure, outside the submitted work. AW reports other from AtriCure, outside the submitted work. The authors have no other conflicts of interest to declare.

Ethical Statement: The authors are accountable for all aspects of the work in ensuring that questions related to the accuracy or integrity of any part of the work are appropriately investigated and resolved.

Open Access Statement: This is an Open Access article distributed in accordance with the Creative Commons Attribution-NonCommercial-NoDerivs 4.0 International License (CC BY-NC-ND 4.0), which permits the noncommercial replication and distribution of the article with the strict proviso that no changes or edits are made and the original work is properly cited (including links to both the 
formal publication through the relevant DOI and the license). See: https://creativecommons.org/licenses/by-nc-nd/4.0/.

\section{References}

1. Kirchhof P, Benussi S, Kotecha D, et al. 2016 ESC guidelines for the management of atrial fibrillation developed in collaboration with EACTS. Eur Heart J 2016;37:2893-962.

2. Dukes JW, Marcus GM. Atrial fibrillation begets myocardial infarction. JAMA Intern Med 2014;174:5-7.

3. Badhwar V, Rankin JS, Damiano RJ Jr, et al. The Society of Thoracic Surgeons 2017 Clinical Practice Guidelines for the Surgical Treatment of Atrial Fibrillation. Ann Thorac Surg 2017;103:329-41.

4. Romero JR, Wolf PA. Epidemiology of Stroke: Legacy of the Framingham Heart Study. Glob Heart 2013;8:67-75.

5. Jahangir A, Lee V, Friedman PA, et al. Long-term progression and outcomes with aging in patients with lone atrial fibrillation: a 30-year follow-up study. Circulation 2007;115:3050-6.

6. Chugh SS, Blackshear JL, Shen WK, et al. Epidemiology and natural history of atrial fibrillation: clinical implications. J Am Coll Cardiol 2001;37:371-8.

7. Benjamin EJ, Wolf PA, D'Agostino RB, et al. Impact of atrial fibrillation on the risk of death: the Framingham Heart Study. Circulation 1998;98:946-52.

8. Connolly SJ, Camm AJ, Halperin JL, et al. Dronedarone in high-risk permanent atrial fibrillation. $\mathrm{N}$ Engl J Med 2011;365:2268-76.

9. Vorperian VR, Havighurst TC, Miller S, et al. Adverse effects of low dose amiodarone: a meta-analysis. J Am Coll Cardiol 1997;30:791-8.

10. Sairaku A, Yoshida Y, Ando M, et al. A head-to-head comparison of periprocedural coagulability under anticoagulation with rivaroxaban versus dabigatran in patients undergoing ablation of atrial fibrillation. Clin Drug Investig 2013;33:847-53.

11. Cox JL, Schuessler RB, D'Agostino HJ Jr, et al. The surgical treatment of atrial fibrillation: III. Development of a definitive surgical procedure. J Thorac Cardiovasc Surg 1991;101:569-83.

12. Cox JL, Boineau JP, Schuessler RB, et al. Electrophysiologic basis, surgical development, and clinical results of the maze procedure for atrial flutter and atrial fibrillation. Adv Card Surg 1995;6:1-67.

13. Cox JL. Atrial fibrillation II: Rationale for surgical treatment. J Thorac Cardiovasc Surg 2003;126:1693-9.
14. Muneretto C, Bisleri G, Bontempi L, et al. Durable staged hybrid ablation with thoracoscopic and percutaneous approach for treatment of long- standing atrial fibrillation: a 30-month assessment with continuous monitoring. J Thorac Cardiovasc Surg 2012;144:1460-5; discussion 1465.

15. Ruaengsri C, Schill MR, Khiabani AJ, et al. The Coxmaze IV procedure in its second decade: still the gold standard? European Journal of Cardio-Thoracic Surgery 2018;53:119-25.

16. Ad N, Henry L, Friehling T, et al. Minimally invasive stand-alone Cox-maze procedure for patients with nonparoxysmal atrial fibrillation. Ann Thorac Surg 2013;96:792-8; discussion 798-9.

17. Haïssaguerre M, Jaïs $P$, Shah DC, et al. Spontaneous initiation of atrial fibrillation by ectopic beats originating in the pulmonary veins. N Engl J Med 1998;339:659-66.

18. Ad N, Henry L, Hunt S. The impact of surgical ablation in patients with low ejection fraction, heart failure, and atrial fibrillation. Eur J Cardiothorac Surg 2011;40:70-6.

19. Letsas KP, Efremidis M, Charalampous C, et al. Current ablation strategies Pract 2011;2011:376969.

20. Nyong J, Amit G, Adler AJ, et al. Efficacy and safety of ablation for people with non-paroxysmal atrial fibrillation. Cochrane Database Syst Rev 2016;11:CD012088.

21. Berger WR, Meulendijks ER, Limpens J, et al. Persistent atrial fibrillation: A systematic review and meta-analysis of invasive strategies. Int J Cardiol 2019;278:137-43.

22. Weimar T, Vosseler M, Czesla M, et al. Approaching a paradigm shift: endoscopic ablation of lone atrial fibrillation on the beating heart. Ann Thorac Surg 2012;94:1886-92.

23. European Heart Rhythm Association, European Association for Cardio- Thoracic Surgery, Camm AJ, et al. Guidelines for the management of atrial fibrillation: the Task Force for the Management of Atrial Fibrillation of the European Society of Cardiology (ESC). Eur Heart J 2010;31:2369-429.

24. Fuster V, Rydén LE, Cannom DS, et al. ACC/AHA/ ESC 2006 Guidelines for the Management of Patients with Atrial Fibrillation: a report of the American College of Cardiology/American Heart Association Task Force on Practice Guidelines and the European Society of Cardiology Committee for Practice Guidelines (Writing Committee to Revise the 2001 Guidelines for the Management of Patients With Atrial Fibrillation): developed in collaboration with the European Heart Rhythm Association and the Heart Rhythm Society. 
Circulation 2006;114:e257-354.

25. Oral H, Chugh A, Good E, et al. Radiofrequency catheter ablation of chronic atrial fibrillation guided by complex electrograms. Circulation 2007;115:2606-12.

26. Suwalski P, Suwalski G, Wilimski R, et al. Minimally invasive off-pump video-assisted endoscopic surgical pulmonary vein isolation using bipolar radiofrequency ablation - preliminary report. Kardiol Pol 2007;65:370-4; discussion 375-6.

27. Gerosa G, Bianco R, Buja G, et al. Totally endoscopic robotic-guided pulmonary veins ablation: an alternative method for the treatment of atrial fibrillation. Eur J Cardiothorac Surg 2004;26:450-2.

28. Kiser AC, Wimmer-Greinecker G, Chitwood WR. Totally extracardiac Maze procedure performed on the beating heart. Ann Thorac Surg 2007;84:1783-5.

29. Wolf RK, Schneeberger EW, Osterday R, et al. Videoassisted bilateral pulmonary vein isolation and left atrial appendage exclusion for atrial fibrillation. J Thorac Cardiovasc Surg 2005;130:797-802.

30. Saltman AE, Rosenthal LS, Francalancia NA, et al. A completely endoscopic approach to microwave ablation for atrial fibrillation. Heart Surg Forum 2003;6:E38-41.

31. Bisleri G, Bottio T, Manzato A, et al. Surgical treatment of lone atrial fibrillation in an awake patient. Heart Surg Forum 2005;8:E158-60.

32. Muneretto C, Bisleri G, Bontempi L, et al. Successful treatment of lone persistent atrial fibrillation by means of a hybrid thoracoscopic- transcatheter approach. Innovations (Phila) 2012;7:254-8.

33. Edgerton JR, Jackman WM, Mack MJ. A new epicardial lesion set for minimal access left atrial maze: the Dallas lesion set. Ann Thorac Surg 2009;88:1655-7.

34. Boersma LV, Castella M, van Boven W, et al. Atrial fibrillation catheter ablation versus surgical ablation treatment (FAST): a 2-center randomized clinical trial. Circulation 2012;125:23-30.

35. Castellá M, Kotecha D, van Laar C, et al. Thoracoscopic vs. catheter ablation for atrial fibrillation: long-term follow-up of the FAST randomized trial. Europace 2019;21:746-53.

36. Phan K, Phan S, Thiagalingam A, et al. Thoracoscopic surgical ablation versus catheter ablation for atrial fibrillation. Eur J Cardiothorac Surg 2016;49:1044-51.

37. Zembala MO, Suwalski P. Minimally invasive surgery for atrial fibrillation. J Thorac Dis 2013;5:S704-12.

38. Mahapatra S, LaPar DJ, Kamath S, et al. Initial experience of sequential surgical epicardial-catheter endocardial ablation for persistent and long-standing persistent AF with long-term follow-up. Ann Thorac Surg 2011;91:1890-8.

39. Pison L, La Meir M, van Opstal J, et al. Hybridthoracoscopic surgical and transvenous catheter ablation of AF. J Am Coll Cardiol 2012;60:54-61.

40. Nanthakumar K, Plumg VJ, Epstein A et al. Resumption of Electrical Conduction in Previously Isolated Pulmonary Veins Rationale for a Different Strategy? Circulation 2004;109:1226-9.

41. Bulava A, Mokracek A, Kurfirst V. Delayed Electroanatomic Mapping After Surgical Ablation for Persistent Atrial Fibrillation. Ann Thorac Surg 2017;104:2024-9.

42. de Asmundis C, Chierchia GB, Mugnai G, et al. Midterm clinical outcomes of concomitant thoracoscopic epicardial and transcatheter endocardial ablation for persistent and long-standing persistent atrial fibrillation: a single-centre experience. Europace 2017;19:58-65.

43. La Meir M, Gelsomino S, Lucà F, et al. Minimally invasive thoracoscopic hybrid treatment of lone atrial fibrillation: early results of monopolar versus bipolar radiofrequency source. Interact Cardiovasc Thorac Surg 2012;14:445-50.

44. Zembala M, Filipiak K, Kowalski O, et al. Minimally invasive hybrid ablation procedure for the treatment of persistent atrial fibrillation: one year results. Kardiol Pol 2012;70:819-28.

45. Gersak B, Pernat A, Robic B, et al. Low rate of atrial fibrillation recurrence verified by implantable loop recorder monitoring following a convergent epicardial and endocardial ablation of atrial fibrillation. J Cardiovasc Electrophysiol 2012;23:1059-66.

46. Kiser AC, Landers M, Horton R, et al. The convergent procedure: a multidisciplinary atrial fibrillation treatment. Heart Surg Forum 2010;13:E317-21.

47. Badhwar N, Al-Dosari G, Dukes J, et al. Subxiphoid Hybrid Approach for Epicardial/Endocardial Ablation and LAA Exclusion in Patients with Persistent and Longstanding Atrial Fibrillation. J Atr Fibrillation 2018;11:2014.

48. Geršak B, Zembala MO, Müller D, et al. European experience of the convergent atrial fibrillation procedure: Multicenter outcomes in consecutive patients. J Thorac Cardiovasc Surg 2014;147:1411-6.

49. Salenger R, Lahey SJ, Saltman AE. The completely endoscopic treatment of atrial fibrillation: report on the first 14 patients with early results. Heart Surg Forum 2004;7:E555-8. 
50. Navaratnarajah M, Luthra S, Ohri S. Chapter 6: Surgical Treatment of Atrial Fibrillation. Intech Open, 2020.

51. Steinberg JS, Varma N, Cygankiewicz I, et al. 2017 ISHNE-HRS expert consensus statement on ambulatory ECG and external cardiac monitoring/telemetry. Heart Rhythm 2017;14:e55-96.

52. Galli A, Ambrosini F, Lombardi F. Holter Monitoring and Loop Recorders: From Research to Clinical Practice. Arrhythm Electrophysiol Rev 2016;5:136-43.

53. Dunning J, Nagendran M, Alfieri OR, et al. Guideline

Cite this article as: Witkowska A, Suwalski P. Insights from advancements and pathbreaking research on the minimally invasive treatment of atrial fibrillation. J Thorac Dis 2021;13(3):2000-2009. doi: 10.21037/jtd-20-1876 for the surgical treatment of atrial fibrillation. Eur J Cardiothorac Surg 2013;44:777-91.

54. Witkowska A, Smoczyński RH, Drobiński D, et al. Left atrial appendage stapling during totally thoracoscopic ablation of long-standing lone persistent atrial fibrillation - lessons learnt. Kardiochirurgia i Torakochirurgia Polska 2013;10:330-3.

55. Salzberg SP, Plass A, Emmert MY, et al. Left atrial appendage clip occlusion: early clinical results. J Thorac Cardiovasc Surg 2010;139:1269-74. 